
\title{
R Reserach S Suare \\ Effect of Elective Induction of Labor on Pregnancy Outcome in Low-Risk Pregnant Women At or Beyond Later Than Term
}

\section{Qinjian Zhang}

Fujian Medical University

\section{Siwen Chen}

Fujian Medical University

\section{Xia Xu}

Fujian Women and Children Hospital

Huale Zhang

Fujian Women and Children Hospital

Jianying Yan ( $\nabla$ yanjy2019@fjmu.edu.cn )

Fujian Women and Children Hospital

\section{Research Article}

Keywords: Later than term, Low-risk pregnancy, Elective induction of labor, Postpartum hemorrhage

Posted Date: December 1st, 2020

DOI: https://doi.org/10.21203/rs.3.rs-112268/v1

License: (c) (1) This work is licensed under a Creative Commons Attribution 4.0 International License. Read Full License 


\section{Abstract}

Background Singleton low-risk pregnant women without obstetric or medical complications,can expect to terminate pregnancy by selective induced labor until 41 weeks later.But recent researches showed it is reasonable to offer elective induction of labor to low-risk nulliparous women 39 weeks 0 days of gestation, and this recommend need further research be conducted to measure the impact of this practice.The fundamental question is whether this operation will increase the additional risk of mother and fetus.

Objective To investigate the effect of induced labor on maternal and fetal pregnancy outcome.

Methods The method of this study is a retrospective case-control study.In this study, a total of 4386 pregnant women with singleton low-risk who underwent regular prenatal examination and successful vaginal delivery at or more than 41 weeks 0 days of gestation in Fujian Maternal and Child Health Hospital from January 2014 to December 2018. Review the clinical data,according to the mode of labor initiation, they were divided into induced labor group (2007 cases) and spontaneous onset of labor group (2361 cases). Further stratified analysis was carried out according to age and parity. Two-sample independent $t$-test and $\chi^{2}$ test were used to analyze the differences of clinical characteristics such as maternal age and parity between the two groups. Logistic regression model was used to analyze the effect of induced labor on pregnancy outcome.

Results The total duration of labor in the induced labor group was significantly longer than that in the spontaneous onset of labor group [ $(9.37 \pm 5.37) v s(8.82 \pm 5.13) \mathrm{h}, P \otimes 0.001]$, associated with more postpartum blood loss[ $(219.18 \pm 188.32) v s(199.95 \pm 124.69) \mathrm{mL}, P=0.01]$, and the incidence of severe postpartum hemorrhage(sPPH) was significantly higher[0.8\%(16/2007) vs $0.33 \%(8 / 2361), P$ $=0.041]$;increase but no significant difference in the incidence of postpartum hemorrhage(PPH) $[3.8 \%$ $(77 / 2007)$ vs $2.8 \%(66 / 2361), P=0.054]$. However,operative vaginal birth was more common in the induced labor group[3.8\%(77/2007) vs 2.8\% (66/2361), $P=0.054]$.Compared with spontaneous onset of labor,NICU admissions were higher with induction[3.8\%(77/2007) vs $2.8 \%(66 / 2361), P=0.054]$;but not significantly increase the risk of the third degree amniotic fluid contamination,placental abruption,birth weight and its distribution, sex of newborn, incidence of neonatal brain injury, incidence of neonatal intraventricular hemorrhage, perinatal death, neonatal hyperbilirubinemia, neonatal septicemia, neonatal pneumonia and Apgar $\leq 7$ at birth $(P \otimes 0.05)$. After adjusting for age, the operation of induced labor in nulliparous is more likely to occur $\mathrm{PPH}[2.74 \%(55 / 2007)$ vs $1.65 \%$ (39/2361); $R R=1.557 ; 95 \% C l: 1.039 \sim 2.332, P \otimes 0.05]$.

Conclusion Selective induction of labor increases the complications of mother and child.Low-risk pregnant women should try to avoid induction without medical indications.

\section{Introduction}


Elective induction of labor ((IOL) means that before the spontaneous onset of labor, the labor process is initiated by human stimulation of uterine contraction to achieve the purpose of delivery, often when the risks of continuing pregnancy outweigh the benefits. As a routine obstetrical operation, the proportion of induced labor is about $4 \% \sim 12 \%$ in developing countries and more common in developed countries, including 26\% in Australia, 23.3\% in the United Kingdom and the United States. The proportion of induced labor reported by Queensland in 2014 was 24.9\% [1]. The risk of mother and fetus increases in the third trimester of pregnancy,as one of the most important means of termination of pregnancy, rational use of $\mathrm{IOL}$ can maximize the outcome of pregnancy.

Pregnancies progressed to 41 weeks with the risks of adverse pregnancy outcomes increased,and the perinatal morbidity and mortality increased significantly after 42 weeks of pregnancy.It is recommended that the gestational age of delivery should be less than 42 weeks [2]. Low-risk pregnant women is recommended the pregnancy be terminated between 41 and 42 weeks of pregnancy,it is means that women with gestational weeks $\geq 41$ weeks without spontaneous parturition should be treated with $\mathrm{IOL}[3]$.

A large multicenter randomized controlled study from 2014 to 2017 found that low-risk primigravida with IOL after 39 weeks could avoid one cesarean section for every 28 induced deliveries,had a lower risk of perinatal death and severe neonatal complications than continued expectant pregnancy[4].Based on this study, Society of Maternal-Fetal(SMFM) recommends it is reasonable to offer elective induction of labor to low-risk nulliparous women after 39 weeks 0 days of gestation who check the gestational age accurately[2].In fact, spontaneous onset of labor is not a clinical "decision",during the process of waiting for spontaneous onset of labor, complications may occur and reduce the possibility of vaginal delivery, so it is not appropriate to compare waiting for spontaneous onset of labor with IOL. So the evidence-based evidence on which previous recommendations are based may require better research to reconsider.

To answer the fundamental question of whether it is safe to relax the indication of induced labor is whether operation of induced labor will increase the additional risk of mother and fetus. This study retrospectively reviewed the clinical data from low-risk pregnant women with later than term and successful vaginal delivery, reduces the influence of confounding factors such as pregnancy complications, minimizes the difference between the two groups, and discusses the influence of IOL on the pregnancy outcome of low-risk pregnant women.

\section{Methods}

\section{Study design and data source}

A retrospective cohort study was conducted a total of 4386 pregnant women with singleton low-risk who underwent regular prenatal examination and successful vaginal delivery at or more than 41 weeks 0 days of gestation between January 2014 to December 2018 in Fujian Maternal and Child Health Hospital, which is an affiliated hospital of Fujian Medical University.According to the mode of labor initiation, they were divided into induced labor group (2007 cases) and spontaneous onset of labor group (2361 cases). 
This retrospective study accordance with relevant Declaration of Helsinki guidelines and regulations. Written informed consent is not required because the data are anonymous and do not affect the patient's treatment, and ethical approval for this study has not been granted ethics committee approval prior to commencing due to retrospective nature, informed consent waived from granted by the ethics committee of Fujian Provincial Maternity and Children's Hospital.

\section{Inclusion and exclusion criteria}

Women who were age between 16 and 45 years old and gave vaginal birth after 41 weeks 0 days, with reliable dating and no obstetric or medical complications were eligible, regardless of favorability of cervical examination. Women were excluded if with scar uterus and transferred to cesarean section.Records with missing data on gestational age and the amount of vaginal bleeding at the time of delivery were excluded.

\section{Exposure, outcome and other variables of interest}

A pregnant women is 'full term' if born anytime from 37 to 42 weeks, then further divides the gestational age into 'early term', between 37 weeks and 38 weeks 6 days; 'full term', between 39 weeks and 40 weeks 6 days; 'late term', the 41 st week; 'post term', after 42 weeks. The gestational age is verified by the crown rump length measured by ultrasound in early pregnancy. If the estimated gestational week difference is more than 5 days when the pregnancy is less than 9 weeks, or more than 7 days between 9 and 14 weeks of pregnancy, the gestational week determined by ultrasound shall prevail.The amount of vaginal bleeding within 24 hours after vaginal birth up to or more than $500 \mathrm{~mL}$ is postpartum hemorrhage (PPH), bleeding volume $\geq 1000 \mathrm{~mL}$ is severe postpartum hemorrhage (sPPH).

\section{Statistical analyses}

Two-sample independent $t$-test and $\chi 2$ test were used to analyze the differences of clinical characteristics such as maternal age and parity between the two groups. Logistic regression model was used to analyze the effect of induced labor on pregnancy outcome,and the outcomes were reported as mean differences

( $M D$; for continuous outcomes) or odds ratios ( $O R$; for binary outcomes), with their accompanying $95 \%$ confidence intervals $(C l)$.

\section{Results}

There were hospital births between January 2014 to December 2018 in Fujian Maternal and Child Health Hospital. After excluding records of women who

did not meet our study criteria ( $\mathrm{N}=44020)$, and those who had Obstetric or medical complications $(\mathrm{N}=331), 4386$ pregnant women with singleton low-risk who successful vaginal delivery at 
or more than 41 weeks 0 days of gestation were included,hey were divided into induced labor group (2007 cases) and spontaneous onset of labor group (2361 cases) (Fig. 1).

\section{Baseline characteristics}

The pregnancy length in IOL group $(289.26 \pm 1.54 \mathrm{~d})$ was longer than that in spontaneous onset of labor group ( $288.33 \pm 1.62 \mathrm{~d}$ ), and with more nulliparous( $71.0 \%$ and $66.6 \%$, respectively),the difference was statistically significant.The median maternal age was 28 years,there was no significant difference in average age and distribution of each age range and between the two groups ( $P>0.05)$. Additional baseline characteristics of all study participants are reported in Table 1.

\section{Objective 1: Correlation between the duration of labor and induction of labor}

The total duration of labor and the duration of each stage of labor in the IOL group were longer than those in the spontaneous onset of labor group,and there were significant differences between the two groups in the first $[(8.62 \pm 5.12) v s(8.17 \pm 4.88) \mathrm{h}]$ and second stages $[(0.65 \pm 0.78) v s(0.55 \pm 0.69) \mathrm{h}]$ of labor and the total duration of labor[ $(9.37 \pm 5.37) v s(8.82 \pm 5.13) \mathrm{h}](P<0.05)$.Additional characteristics of duration of labor are reported in Table 2.

The total duration of labor in the IOL group was shortened with the women who multipara,and the total duration of labor in nulliparous was the longest (11.07 $\pm 5.12 \mathrm{~h}$ ), and the multipara was mostly within 8 hours, with increase of the number of parturition,the duration of labor is gradually shortened.

\section{Objective 2: Correlation between induction of labor and postpartumhemorrhage}

The amount of vaginal bleeding increased with the prolongation of the total duration of labor, and increased significantly when the total stage of labor was $\geq 20$ hours. The IOL group with induced labor intervention associated with more postpartum blood loss[ (219.18 \pm 188.32$) v s(199.95 \pm 124.69)$ $\mathrm{mL}, P=0.01]$.Moreover,the amount of vaginal bleeding was more when compared with the every same total duration of labor with spontaneous onset of labor group,and the incidence of sPPH was significantly higher[0.8\%(16/2007) vs 0.33\% (8/2361), $P=0.041]$;but no significant difference in the incidence of PPH [3.8\%(77/2007)vs2.8\% (66/2361), $P=0.054]$. (Fig.2).

The IOL operation for nulliparous women was more likely to occur PPH (1.65\% vs $2.74 \%, R R: 1.557 \%, 95 \%$ Cl:1.039 2.332) compared with spontaneous onset of labor group.However,among the multipara, there was no significant difference in the incidence of PPH between the two groups[ $(P>0.05)$ Table 3].

\section{Objective 3: Correlation between induction of labor and other secondary pregnancy outcome}

The incidence of chorioamnionitis in the IOL group was significantly higher than that in the spontaneous onset of labor group ( $4.33 \%$ vs $2.33 \%, P \otimes 0.05)$, and so as the rate of operative vaginal birth $(3.5 \%$ vs 5.0 $\%, P \otimes 0.05)$. There was no significant difference in the incidence of third degree amniotic fluid 
contamination ( $15.5 \%$ vs $14.7 \%, P \otimes 0.05)$ and placental abruption ( $4.48 \%$ vs $2.96 \%, P \Downarrow 0.05)$ between the two groups.

The NICU admission rate of newborns in IOL group was significantly higher than that in spontaneous onset of labor ( $3.74 \%$ vs $2.16 \%, P \otimes 0.05)$. However, there was no significant difference between the two groups in birth weight and its distribution,sex of newborn, incidence of neonatal brain injury, incidence of neonatal intraventricular hemorrhage,perinatal death,neonatal hyperbilirubinemia,neonatal septicemia, neonatal pneumonia and Apgar $\leq 7$ at birth $(P \otimes 0.05)$.

\section{Discussion}

The maternal and fetal risk increases in the third trimester of pregnancy, and IOL can be used as one of the important measure to terminate pregnancy when the benefits of termination of pregnancy are greater than those of continued pregnancy. Correctly grasping the timing of induced labor and reasonably choosing the mode of induced labor can optimize the pregnancy outcome to the maximum extent. At present, the results of the study on the effect of IOL on pregnancy outcome in low-risk pregnant women are still controversial, which may be related to the selection of research objects and research design. This study adopted a retrospective case-control study, selected low-risk delayed pregnancy and vaginal delivery as the research object, compared and analyzed the pregnancy outcome of IOL and spontaneous onset of labor, reduced the differences between groups and confounding factors, and the results were more objective, which provided the basis for the choice of delivery decision.

Vahratian et al.[5] showed that the labor process of pregnant women with IOL was significantly longer than that of pregnant women in spontaneous onset, which was consistent with the results of this study. The results showed that the total duration of labor and the duration of each stage of labor in the IOL group were more than those in the spontaneous onset labor group. The increase in the duration of labor in the IOL group was mainly in the first stage of labor, and the first stage of labor and the total stage of labor were increased by about $0.5 \mathrm{~h}$. Further stratified analysis of parity showed that the total duration of labor in IOL group was shortened with the increase of parity, and the total duration of labor was the longest when nulliparous women were operated with IOL (11.07 $\pm 5.12 \mathrm{~h}$ ), by contrast the total duration of labor of multipara is usually less than 8 hours. The percentage of multipara women in spontaneous onset of labor group was higher than that in IOL group (33.4\% vs $29.0 \%$ ), which suggested that multipara women were more likely to start labor spontaneously and the duration of labor was shorter.

Cheng et al. [6] found that the extension of the first stage of labor will increase prolongation of the second stage of labor,and is associated with an increased risk of PPH[7-8]. It is suggested that the prolongation of labor time may cause uterine weakness in the third stage of labor, thus increasing the risk of PPH. The results of this study showed that the postpartum blood loss in the IOL group was more than that in the spontaneous onset of labor group [(219.18 \pm 188.32$) v s(199.95 \pm 124.69) \mathrm{mL}]$. The average postpartum blood loss increased with the prolongation of the total stage of labor, and the postpartum blood loss in the total stage of labor $\geq 20$ hours increased significantly. It is suggested that the amount 
of postpartum blood loss in the IOL group is higher than that in the spontaneous onset of labor group due to the prolongation of the course of labor, and the lead to the incidence of PPH and sPPH,especially in the nulliparous women because of the significantly longer total duration of IOL. Therefore, when IOL is performed on nulliparous women, which could serve as a clinical warning indicate a higher incidence of atonic $\mathrm{PPH}$.

The results of this study showed that the amount of vaginal bleeding in the IOL group with induced labor intervention associated with more postpartum blood loss when compared with the every same total duration of labor in spontaneous onset of labor group, suggesting that prolonged labor is not the only reason for the increase in postpartum blood loss. IOL operation can lead to an increase in postpartum blood loss, which shows that the peak of postpartum blood loss in IOL group moves forward with the duration of labor(Fig.2).

Recent studies have shown that [9-10], compared with spontaneous onset labor parturition, IOL does not lead to an increase in adverse maternal and perinatal outcomes.Therefore, the impact of active labor induction of low-risk pregnant women on the clinical outcome of parturients and perinatal infants is not clear.This study showed that there was no significant difference in the incidence of neonatal brain injury, neonatal intraventricular hemorrhage, perinatal death, neonatal hyperbilirubinemia, neonatal septicemia, neonatal pneumonia and Apgar $\leq 7$ between the IOL group and control group. However, the risk of admission to NICU in IOL group is higher than that in spontaneous onset of labor, which may be related to the higher incidence of operative vaginal birth and chorioamnionitis in IOL group.

The difference between the results of this study and previous studies may be related to the choice of research objects. Previous studies aimed at all populations, increased the risk of adverse pregnancy outcomes with the progression of gestational weeks, increased the risk of selective cesarean section and emergency cesarean section, and affected the comparison of perinatal outcomes[11-12]. In this study, pregnant women with low risk, later than term means no obstetric or medical complications were eligible and with successful vaginal delivery were selected as the study population to eliminate the influence of confounding factors to the maximum extent. The results can better reflect the impact of IOL operation on perinatal outcome.

\section{Conclusions}

To sum up, a total duration of labor is prolonged and increase the postpartum blood loss in IOL group,increases the complications of maternal and perinatal.The clinical decision-making of IOL operation without medical indication for low-risk nulliparous needs to be more prudent,low-risk pregnant women should try to avoid induction without medical indications.

\section{Declarations}

\section{Statement}


This retrospective study accordance with relevant Declaration of Helsinki guidelines and regulations.

\section{Ethics approval and consent to participate}

This retrospective study accordance with relevant Declaration of Helsinki guidelines and regulations. Written informed consent is not required because the data are anonymous and do not affect the patient's treatment, and ethical approval for this study has not been granted ethics committee approval prior to commencing due to retrospective nature, informed consent waived from granted by the ethics committee of Fujian Provincial Maternity and Children's Hospital.

Permission to access the anonymized (non-identified) data was granted by the Fujian Provincial Maternity and Children's Hospital Database steering committee.

\section{Consent for publication}

All data was anonymized, therefore individual consent for publication was not required.

\section{Availability of data and materials}

Data were anonymized and no patient identifying information was included for preserve patient confidentiality. All data to evaluate the conclusions in the paper available for scientific purposes if needed.All data generated or analysed during this study are included in this published article and its supplementary information files.

\section{Competing interests}

The authors declare that they have no competing interest.

\section{Funding}

This study was approved by National Health and Family Planning Commission Science Foundation(2019-WJ-04), the Fujian Science and Technology Project (2018Y0005), and the Guide Fund for the Development of Local Science and Technology from the Central Government (2020L3019).

\section{Author Contributions Statement}

Zhang Qinjian And Yan Jianying conceptualised the study. Chen Siwen, Xu Xia and Zhang Huale contributed to data acquisition. Zhang Qinjian and Chen Siwen designed the analyses, Zhang Qinjian drafted the manuscript. All authors revised the manuscript for important intellectual content.

\section{Acknowledgements}

Not applicable.

\section{References}


1. Osterman $\mathrm{M} \mathrm{J}$, Martin $\mathrm{J} A$. Recent declines in induction of labor by gestational age[J]. NCHS data brief, 2014, 155(155):1-8.

2. Society of Maternal-Fetal (SMFM) Publications Committee. Electronic address: pubs@smfm.org,SMFM Statement on Elective Induction of Labor in Low-Risk Nulliparous Women at Term: the ARRIVE Trial.[J] .Am. J. Obstet. Gynecol., 2019, 221: B2-B4.

3. WHO recommendations: induction of labour at or beyond term. Geneva: World Health Organization; 2018. Licence: CC BY-NC-SA 3.0 IGO.ISBN 978-92-4-155041-3.

4. Grobman William A,Rice Madeline M,Reddy Uma $M$ et al. Labor Induction versus Expectant Management in Low-Risk Nulliparous Women.[J] .N. Engl. J. Med., 2018, 379: 513-523.

5. Vahratian Anjel,Zhang Jun,Troendle James $F$ et al. Labor progression and risk of cesarean delivery in electively induced nulliparas.[J] .Obstet Gynecol, 2005, 105: 698-704.

6. Cheng YW, Delaney SS, Hopkins LM, Caughey AB. The association between the length of first stage of labor, mode of delivery, and perinatal outcomes in women undergoing induction of labor. Am J Obst Gynecol. 2009; 201:477 e1-7.

7. Cheng YW, Hopkins LM, Laros RK Jr., Caughey AB. Duration of the second stage of labor in multiparous women: maternal and neonatal outcomes. Am J Obst Gynecol. 2007; 196:585 e1-6.

8. Janni W, SchiessI B, Peschers U, Huber S, Strobl B, Hantschmann P, et al. The prognostic impact of a prolonged second stage of labor on maternal and fetal outcome. Acta Obstet Gynecol Scand. 2002;81:214-21.

9. Wennerholm Ulla-Britt,Saltvedt Sissel,Wessberg Anna et al. Induction of labour at 41 weeks versus expectant management and induction of labour at 42 weeks (SWEdish Post-term Induction Study, SWEPIS): multicentre, open label, randomised, superiority trial.[J] .BMJ, 2019, 367: 16131.

10. Souter V , Painter I , Sitcov K, et al. Maternal and newborn outcomes with elective induction of labor at term[J]. American Journal of Obstetrics and Gynecology, 2019, 220(3):273.e1-273.e11.

11. Brane E, Olsson A, Andolf E. A randomized controlled trial on early induction compared to expectant management of nulliparous women with prolonged latent phases. Acta Obstet Gynecol Scand. 2014;93(10):1042-1049.

12. Khireddine I , Ray C L , Dupont C , et al. Induction of Labor and Risk of Postpartum Hemorrhage in Low Risk Parturients[J]. PLOS ONE, 2013, 8.

\section{Tables}

Table 1 Baseline characteristics and main pregnancy outcomes 


\begin{tabular}{|c|c|c|c|}
\hline \multirow[t]{2}{*}{ Baseline characteristics } & $\begin{array}{l}\text { Spontaneous onset of labor } \\
\bigotimes \mathrm{N}=2361 \rrbracket\end{array}$ & $\begin{array}{l}\text { Induction of labor } \\
\varangle \mathrm{N}=2007 \rrbracket\end{array}$ & \multirow{2}{*}{$\begin{array}{l}P \\
\text { value }\end{array}$} \\
\hline & $\mathrm{N} \otimes \% \rrbracket$ & $\mathrm{N} \bigotimes \% \bigotimes$ & \\
\hline Maternal age (years) & $28.59 \rrbracket 17 \rrbracket 44 \rrbracket$ & $28.55 \rrbracket 16 \rrbracket 46 \rrbracket$ & 0.732 \\
\hline$\otimes 18$ & $5 \rrbracket 0.2 \% \rrbracket$ & $5 \rrbracket 0.2 \% \rrbracket$ & NC \\
\hline $18-34$ & $2188 \otimes 92.7 \% \rrbracket$ & $1853 \llbracket 92.3 \% \rrbracket$ & NC \\
\hline $35-39$ & $147 \rrbracket 6.2 \% \rrbracket$ & $130 \rrbracket 6.5 \% \rrbracket$ & $\mathrm{NC}$ \\
\hline$\geq 40$ & $21 \bowtie 0.9 \% \rrbracket$ & $19 \bowtie 0.9 \% \rrbracket$ & $\mathrm{NC}$ \\
\hline $\begin{array}{l}\text { Mean gestational age delivery } \\
\text { (days) }\end{array}$ & $288.33 \rrbracket 287 \rrbracket 304 \rrbracket$ & $289.26 \rrbracket 287 \rrbracket 313 \rrbracket$ & 0 \\
\hline \multicolumn{4}{|l|}{ Gravidity } \\
\hline$=1$ & $1163 \llbracket 49.3 \% \bigotimes$ & $1029 \otimes 51.3 \% \rrbracket$ & 0.185 \\
\hline$>1$ & $1198 \rrbracket 50.7 \% \rrbracket$ & $978 \rrbracket 48.7 \% \rrbracket$ & 0.185 \\
\hline \multicolumn{4}{|l|}{ Parity } \\
\hline$=1$ & $1573 \llbracket 66.6 \% \rrbracket$ & $1425 \rrbracket 71.0 \% \rrbracket$ & 0.002 \\
\hline$>1$ & $788 \llbracket 33.4 \% \bigotimes$ & $582 \rrbracket 29.0 \% \rrbracket$ & 0.002 \\
\hline \multicolumn{4}{|l|}{ Maternal outcomes } \\
\hline Operative vaginal birth & $82 \bowtie 3.5 \% \rrbracket$ & $101 \rrbracket 5.0 \% \rrbracket$ & 0.01 \\
\hline Chorioamnionitis & $55 \rrbracket 2.33 \% \rrbracket$ & $87 \rrbracket 4.33 \% \rrbracket$ & 0.0002 \\
\hline placental abruption & $7 \rrbracket 2.96 \% \rrbracket$ & $9 \rrbracket 4.48 \% \rrbracket$ & 0.408 \\
\hline Amniotic fluid contamination & $348 \rrbracket 14.7 \% \rrbracket$ & $291 \rrbracket 14.5 \% \rrbracket$ & 0.823 \\
\hline Postpartum blood loss & $199.95 \rrbracket 70 \rrbracket 2190 \rrbracket$ & $219.18 \bowtie 65 \rrbracket 4560 \rrbracket$ & 0.01 \\
\hline postpartum hemorrhage & $66 \rrbracket 2.8 \% \rrbracket$ & $77 \rrbracket 3.8 \% \rrbracket$ & 0.054 \\
\hline $\begin{array}{l}\text { severe postpartum } \\
\text { haemorrhage }\end{array}$ & 8\0.33\%区 & $16 \rrbracket 0.8 \% \bigotimes$ & 0.041 \\
\hline \multicolumn{4}{|l|}{ Neonatal outcomes } \\
\hline Apgar score at $1 \min (4-7)$ & $3 \rrbracket 0.1 \% \rrbracket$ & $8 \rrbracket 0.4 \% \rrbracket$ & 0.074 \\
\hline Apgar score at $1 \min (\mathbb{8} 4)$ & $1 \otimes 0 \otimes$ & $0 \otimes 0 \bigotimes$ & 0.357 \\
\hline Hyperbilirubinemia & $628 \rrbracket 26.6 \% \rrbracket$ & $583 \llbracket 29 \llbracket 05 \% \rrbracket$ & 0.072 \\
\hline Perinatal death & 0 & 0 & NC \\
\hline Neonatal sepsis & $21 \otimes 0.89 \% \rrbracket$ & $16 \otimes 0.8 \% \square$ & 0.74 \\
\hline
\end{tabular}




\begin{tabular}{|c|c|c|c|}
\hline Neonatal pneumonia & $22 \rrbracket 0.93 \% \rrbracket$ & 16 & 0.633 \\
\hline Neonatal brain injury & $1 \otimes 0 \rrbracket$ & $0 \otimes 0 \bigotimes$ & 0.357 \\
\hline $\begin{array}{l}\text { Neonatal intracranial } \\
\text { hemorrhage }\end{array}$ & $2 \rrbracket 0.09 \% \rrbracket$ & $1 \otimes 0.05 \% \bigotimes$ & 0.66 \\
\hline NICU admission & $51 \rrbracket 2.16 \% \rrbracket$ & 75囚3.74\%区 & 0.002 \\
\hline \multicolumn{4}{|l|}{ Sex of newborn } \\
\hline Male & 1098ه46.51\%邓 & $950 \rrbracket 47.33 \% \rrbracket$ & 0.584 \\
\hline Female & $1263 \rrbracket 53.49 \% \rrbracket$ & $1057 \otimes 52.67 \% \rrbracket$ & 0.584 \\
\hline Birth weight]g] & & & 0.84 \\
\hline$\leq 2500$ & $3 \rrbracket 0.1 \% \rrbracket$ & $3(0.1 \%)$ & 0.21 \\
\hline 2501-3999 & $2169(91.9 \%)$ & $1864(92.9 \%)$ & 0.20 \\
\hline$\geq 4000$ & $189(8.0 \%)$ & $140(7.0 \%)$ & \\
\hline
\end{tabular}

Table 2 Correlation induction of labor with the duration of labor and postpartum blood loss. The total duration of labor and the duration of each stage of labor in the IOL group were longer than those in the spontaneous onset of labor group,and there were significant differences between the two groups in the first $[(8.62 \pm 5.12)$ vs $(8.17 \pm 4.88) \mathrm{h}]$ and second stages[$[(0.65 \pm 0.78)$ vs $(0.55 \pm 0.69) \mathrm{h}]$ of labor and the total duration of labor $[(9.37 \pm 5.37)$ vs $(8.82 \pm 5.13) h](P<0.05)$ 


\begin{tabular}{|c|c|c|c|}
\hline & $\begin{array}{l}\text { Spontaneous onset of labor } \\
\bigotimes N=2361 \rrbracket\end{array}$ & $\begin{array}{l}\text { Indcution of labor } \\
\square \mathrm{N}=2007 \rrbracket\end{array}$ & $\begin{array}{l}\mathrm{P} \\
\text { value }\end{array}$ \\
\hline & $N \otimes \% \bigotimes$ & $N \rrbracket \% \rrbracket$ & \\
\hline \multicolumn{4}{|l|}{ Duration of labor } \\
\hline First stages of labor & $8.17 \pm 4.88$ & $8.62 \pm 5.12$ & \\
\hline Second stages of labor & $0.55 \pm 0.69$ & $0.65 \pm 0.78$ & \\
\hline Third stages of labor & $0.11 \pm 0.11$ & $0.12 \pm 0.12$ & \\
\hline Total stage of labor & $8.82 \pm 5.13$ & $9.37 \pm 5.37$ & \\
\hline \multicolumn{4}{|l|}{ Postpartum blood loss } \\
\hline Postpartum blood loss & $199.95 \rrbracket 70 \rrbracket 2190 \rrbracket$ & $219.18 \rrbracket 65 \rrbracket 4560 \rrbracket$ & 0.01 \\
\hline postpartum hemorrhage & $66 \rrbracket 2.8 \% \rrbracket$ & $77 \rrbracket 3.8 \% \rrbracket$ & 0.054 \\
\hline $\begin{array}{l}\text { severe postpartum } \\
\text { haemorrhage }\end{array}$ & $8 \rrbracket 0.33 \% \rrbracket$ & 16ه0.8\%区 & 0.041 \\
\hline \multicolumn{4}{|l|}{ Parity } \\
\hline$=1$ & $39 \rrbracket 1.65 \% \rrbracket$ & $55 \rrbracket 2.74 \% \rrbracket$ & \\
\hline$>1$ & $27 \rrbracket 1.14 \% 区$ & $4 ه 0.20 \% \rrbracket$ & \\
\hline
\end{tabular}

Table 3 The IOL operation for nulliparous women was more likely to occur PPH compared with spontaneous onset of labor group.However,among the multipara, there was no significant difference in the incidence of PPH between the two groups.IOL=Induction of labor.

\begin{tabular}{|c|c|c|c|c|c|}
\hline \multirow[t]{2}{*}{ Group } & \multirow[t]{2}{*}{ cases } & \multicolumn{4}{|l|}{ Parity } \\
\hline & & 1 & 2 & 3 & $\geq 3$ \\
\hline Spontaneous onset of labor group & 2361 & $39 ه 1.65 \% \rrbracket$ & $25 \rrbracket 1.06 \% \rrbracket$ & $2 \varangle 0.08 \% \rrbracket$ & 0 \\
\hline Induction of labor group & 2007 & $55 \rrbracket 2.74 \% \rrbracket$ & $3 \rrbracket 0.15 \% \rrbracket$ & $1 \rrbracket 0.05 \% \rrbracket$ & 0 \\
\hline$R R \otimes 95 \% \mathrm{Cl} \square$ & - & $1.557 \rrbracket 1.039 \llbracket 2.332 \rrbracket$ & \multicolumn{3}{|c|}{$1.103 \otimes 0.635 \rrbracket 1.917 \rrbracket$} \\
\hline$P$ & - & 0.03 & 0.671 & 0.794 & $N C^{\star}$ \\
\hline
\end{tabular}

\section{Figures}




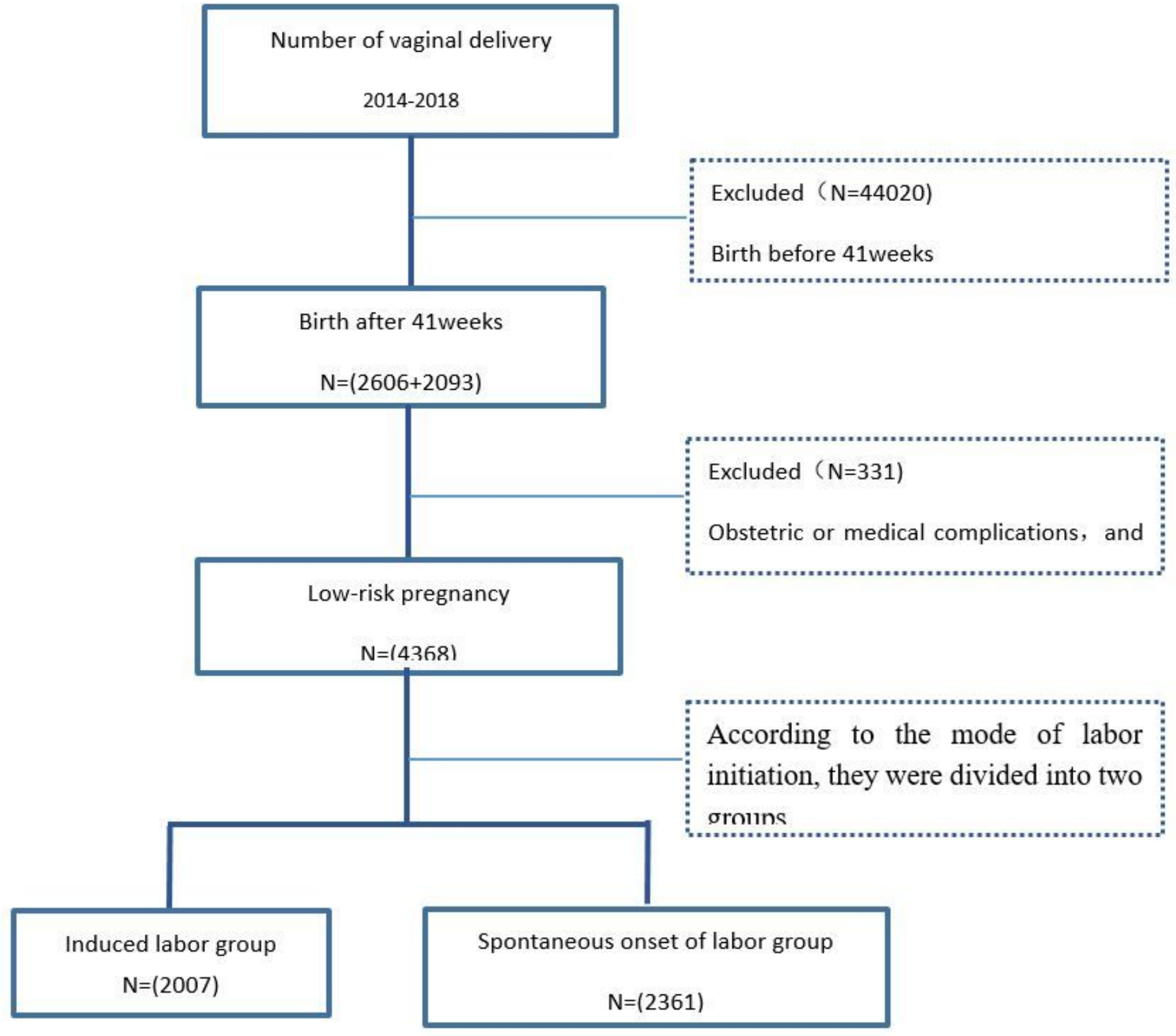

Figure 1

Flow Chart of Subject Selection. VBAC=Vaginal birth after caesarean 


\begin{tabular}{|c|c|c|c|c|c|c|c|}
\hline & \\
\hline
\end{tabular}

\section{Figure 2}

The relationship between the total duration of labor and postpartum blood loss. The postpartum blood loss in IOL group was higher when compared with the every same total duration of labor in spontaneous group.IOL=Induction of labor. 